\title{
Reviewer Acknowledgements for Global Journal of Health Science, Vol. 6, No. 4
}

Global Journal of Health Science wishes to acknowledge the following individuals for their assistance with peer review of manuscripts for this issue. Their help and contributions in maintaining the quality of the journal is greatly appreciated.

Global Journal of Health Science is recruiting reviewers for the journal. If you are interested in becoming a reviewer, we welcome you to join us. Please find the application form and details at http://www.ccsenet.org/reviewer and e-mail the completed application form to gjhs@ccsenet.org.

\section{Reviewers for Volume 6, Number 4}

Ama Pokuaa Fenny, Aarhus University, Denmark

Ammar Eltayeb Ali Hassan, University of Tromsø, Norway

Caroline Elizabeth Bulsara, University of Western Australia, Australia

Donna Wilson, University of Alberta, Canada

Emad Adel Shdaifat, Faculty of Health Sciences, Universiti Teknologi MARA (UiTM), Malaysia

Farahnaz Amini, UCSI University, Malaysia

Fernanda Barbosa Lima-Christian, Federal University of Santa Catarina, Brazil

Francisco Rodenas Rigla, University of Valencia, Spain

Gabriele Messina, University of Siena, Italy

Hadii M Mamudu, East Tennessee State University, United States

Jacob D. Christenson, Mount Mercy University, United States

Kartheek R Balapala, University Tunku Abdul Rahman, Malaysia

Kimberley Geissler, Boston University School of Management, United States

Le Thi Thanh Xuan, Hanoi Medical University, Viet Nam

Loray Daws, British Columbia Masterson Institute, Canada

Manoj K. Mishra, Alabama State University, United States

Masitah Shahrill, Universiti Brunei Darussalam, Brunei Darussalam

Meng Zhao, Texas A\&M University at Corpus Christi, United States

Montarat Thavorncharoensap, Health Intervention and Technology Assessment Program (HITAP), Ministry of Public Health, Thailand

Myo Nyein Aung, Boromrajonani College of Nursing, Thailand

Pavlos Sarafis, Technological Educational Institute Lamia, Faculty of Nursing, Greece

Polly Yeung, Massey University, New Zealand

Qilong Wang, University of Oklahoma, United States

Samira Mansur, Federal University of Santa Catarina, Brazil

Sara Melo, Queen's University Belfast, United Kingdom

Shilpee Singh, University of Pennsylvania, United States

Srikrishna Sulgodu Ramachandra, Public Health Foundation of India, India

Steven Hoffman, University of Texas at San Antonio, United States

Tarek Tawfik Amin, King Faisal University, Saudi Arabia

Tugrul Erbaydar, Ankara University Medical School, Turkey

Yevgeniy Samyshkin, IMS Health, United Kingdom

Zada Pajalic, School of Health and Society, Kristianstad University, Sweden

Zhe Liu, University of Southern California, United States 